\title{
Binding of lipid droplets and mitochondria to intermediate filaments in rat Leydig cells
}

\author{
G. Almahbobi, L. J. Williams, X-G. Han and P. F. Hall \\ Department of Endocrinology, Prince of Wales Hospital, Randwick 2031, NSW, Australia
}

\begin{abstract}
We have examined the distribution of lipid droplets and mitochondria in relation to the cytoskeletons of Leydig cells in primary culture by using light and electron microscopy on living, intact and detergent-extracted cells. After mild extraction with Triton X-100 lipid droplets and mitochondria retained their original distribution within the cell. Double immunofluorescent microscopy showed that both structures co-localise with intermediate filaments. Transmission electron microscopy of intact (unextracted) and mildly extracted Leydig cells showed that intermediate filaments are closely associated with mitochondria and lipid droplets. By examination of stereo pairs, intermediate filaments were shown to establish direct contact with mitochondria and lipid droplets. The association of droplets and mitochondria with intermediate filaments suggests possible mechanisms by which the transport of cholesterol takes place from droplets to mitochondria where this substrate enters the steroidogenic pathway.
\end{abstract}

\section{Introduction}

It is generally agreed that the slow step in steroid synthesis involves the transport of cholesterol from cytoplasmic stores to the inner mitochondrial membrane where steroidogenesis begins with the conversion of cholesterol to pregnenolone (side-chain cleavage) (Hall, 1987). The cytoplasmic stores of cholesterol include lipid droplets that are characteristic of steroidogenic cells (Silberzahn et al., 1985; Almahbobi et al., 1985, 1988; Almahbobi and Hall, 1990). The process of cholesterol transport involves the cytoskeleton and, in particular, actin (Hall et al., 1979; Osawa et al., 1984). The mechanism by which the cytoskeleton promotes this transport is not clear. We have reported that lipid droplets (Almahbobi and Hall, 1990; Almahbobi et al., 1992a) and mitochondria (Almahbobi et al., 1992b) are attached to intermediate filaments in adrenal cells. Since attachment of these structures to intermediate filaments may be important in the regulation of cholesterol transport from droplets to mitochondria, it appeared important to determine whether a similar association is found in other steroidogenic cells. We show here that droplets and mitochondria are bound to intermediate filaments in rat Leydig cells in primary culture.

\section{Materials and Methods}

\section{Materials}

Materials were purchased as follows: Ham's F12, Dulbecco's modified Eagle's medium (DMEM), fetal calf serum (FCS) and horse serum from Flow Laboratories Inc., Irvine; Percoll from Pharmacia LKB, Biotechnology $A B$, Uppsala; Nile red from

Received 29 June 1992
Eastman Kodak Company, Rochester, NY; collagenase type IA, deoxyribonuclease I (DNAase I), BSA, rhodamine 123, immunoglobulin Gs (IgGs) of uninjected animals, goat anti-vimentin and rabbit anti-goat-fluorescein isothiocyanate (FITC) antibodies from Sigma Corporation (St Louis, MO). Mouse antivimentin, sheep anti-mouse-FITC and donkey anti-rabbit-Texas red antibodies were purchased from Amersham International (Buckinghamshire). Goat anti-mouse IgG + IgM antibody conjugated to $5 \mathrm{~nm}$ gold particles was obtained from Janssen (Life Sciences Products, Beerse). Rabbit anti-cytochrome oxidase antibody was a gift from E. Racker, Cornell University, Ithaca, NY. The specificity of this antibody has been described elsewhere (Eytan et al., 1975). All secondary antibodies were affinity purified as stated by suppliers.

\section{Cell isolation and cultures}

Sprague-Dawley rats, aged 30 days, were killed by cervical dislocation. The testes were removed aseptically and immediately transferred into ice cold medium (Ham's F12/DMEM, 1:1). All other procedures were carried out under sterile conditions. Leydig cells were prepared by a published method (Browning et al., 1981) with modification. After decapsulation, testicular tissue was incubated for $30 \mathrm{~min}$ at $37^{\circ} \mathrm{C}$ with gentle shaking in the same medium containing BSA $\left(1 \mathrm{mg} \mathrm{m}^{-1}\right)$, DNAase I $\left(10 \mu \mathrm{g} \mathrm{ml}^{-1}\right)$ and collagenase type I $\left(0.5 \mathrm{mg} \mathrm{ml}^{-1}\right)$. Leydig cells were isolated by means of centrifugation $(800 \mathrm{~g}$ for $20 \mathrm{~min}$ ) through a continuous Percoll gradient. After washing twice, the cells were plated in plastic culture dishes, glass slide chambers or on coverslips. The cultures were maintained in medium supplemented with $12.5 \%$ horse serum, $2.5 \%$ FCS and gentamycin A ( $\left.32 \mathrm{mg} \mathrm{ml}^{-1}\right)$ in a humidified atmosphere of $95 \%$ air: $5 \% \mathrm{CO}_{2}$ for $2-3$ days to obtain cells that were well spread, firmly attached and confluent. The purity of our preparations 
of Leydig cells was greater than $95 \%$ as determined by histoenzymological staining for $3 \beta$-hydroxysteroid dehydrogenase. Production of testosterone with $\left(46 \mathrm{pmol}\right.$ per $10^{6}$ cells $\mathrm{h}^{-1}$, $n=3$ ) and without (24 pmol per $10^{6}$ cells $\mathrm{h}^{-1}, n=3$ ) dibutyryl cAMP was measured by radioimmunoassay after $24 \mathrm{~h}$ of culture, respectively.

\section{Cell extraction}

Cells grown in slide chambers, on coverslips, on gold grids and in dishes were subjected to extraction with buffered Triton X-100 as described by Almahbobi and Hall (1990) and Fey et al. (1984). The concentration of Triton and the duration of extraction were varied. Extraction of Leydig cells with $0.5 \%$ Triton for 5 min gave the best recovery of mitochondria and excellent immunofluorescent staining of all three cellular structures studied (Almahbobi et al., 1992b). In some experiments intermediate filaments were prepared as described by Almahbobi and Hall (1990) and Fey et al. (1984). Intermediate filaments were prepared by extracting cells with $1 \%$ Triton X-100 for 10 min followed by $1 \%$ Triton X-100 containing ammonium sulfate (Fey et al., 1984).

\section{Light microscopy}

Lipid droplets and mitochondria of living cells grown in slide chambers were stained with Nile red (Greenspan et al., 1985; Almahbobi and Hall, 1990) and rhodamine 123 (Johnson et al., 1980; Almahbobi et al., 1992b), respectively. Nile red was used at a concentration of $100 \mathrm{ng} \mathrm{ml}^{-1}$ for $10 \mathrm{~min}$ and rhodamine 123 at $10 \mu \mathrm{g} \mathrm{ml}^{-1}$ for $30 \mathrm{~min}$ at $37^{\circ} \mathrm{C}$. Immunofluorescent staining was performed on extracted cells grown in slide chambers. Cells were fixed in $3.6 \%$ formaldehyde v:v in phosphate buffer saline (PBS, $0.01 \mathrm{moll}^{-1}, \mathrm{pH} 7.2$ ) for $30 \mathrm{~min}$. For double staining of lipid droplets and vimentin-intermediate filaments, mouse anti-vimentin monoclonal antibody (1:5 for $60 \mathrm{~min}$ ) and sheep anti-mouse antibody conjugated to FITC (1:40 for $60 \mathrm{~min}$ ) were used. After extensive washes with PBS, cells were incubated in Nile red as described above. Other details of these procedures have been described elsewhere (Almahbobi and Hall, 1990).

Double indirect immunofluorescence to stain mitochondria and vimentin-intermediate filaments was performed using a modified procedure (Almahbobi et al., 1992b; Almahbobi and Hall, 1993) in which four antibodies (two primary and two secondary) raised in three species were used in the following order: rabbit anti-cytochrome oxidase (1:30 for $60 \mathrm{~min}$ ), wash with PBS, donkey anti-rabbit-Texas red (1:40 for $40 \mathrm{~min}$ ), wash with PBS, goat anti-vimentin (1:20 for $60 \mathrm{~min}$ ), wash with PBS, rabbit anti-goat-FITC (1:20 for $40 \mathrm{~min}$ ), extensive wash with PBS. After this procedure, mitochondria were stained with both fluorochromes, while intermediate filaments were stained only by FITC. Both intermediate filaments and mitochondria are displayed with one light filter (blue); this makes it possible to demonstrate co-localisation of these two structures. However, one of these structures (mitochondria), but not the filaments, was identified by using a second filter (green). A number of control conditions were used for each staining i.e. no primary antibody, no secondary antibody, IgG from uninjected animals and antibody preabsorbed with antigen. The above procedures were all performed at room temperature unless otherwise stated.

\section{Electron microscopy}

Intact and extracted cells grown on coverslips were prepared for conventional electron microscopy as monolayers. Cells were fixed in 1/2 Karnovsky fixative and then processed as described by Almahbobi et al. (1988, 1992a, b). Thin sections were examined with a Hitachi 7000 transmission electron microscope. Stereopair micrographs were made by means of tilting.

\section{Immunoelectron microscopy}

Immunoelectron microscopy was performed on whole mount preparations of intermediate filaments from cells grown on Formvar-coated gold grids. Mouse anti-vimentin antibody was used at a dilution of 1:5 for $60 \mathrm{~min}$ followed by goat anti-mouse antibody conjugated to $5 \mathrm{~nm}$ gold particles (1:25) for $30 \mathrm{~min}$. All other procedures were as described by Almahbobi and Hall (1990). In some experiments post-fixation with osmium tetroxide was omitted. The freeze dried preparations were examined with a Hitachi 7000 transmission electron microscope.

\section{Results}

\section{Light microscopy}

Figure 1 shows living cultured rat Leydig cells ( $a$ and $b$ ) and those extracted with Triton X-100 ( $c$ and d). Lipid droplets are revealed by a specific lipid stain, namely Nile red (Greenspan et al., 1985) and mitochondria are stained by rhodamine 123 which has been widely used to specifically identify mitochondria (Johnson et al., 1980). Lipid droplets appear as spherical structures and in some cases they are arranged in linear array extending from the nucleus (Fig. 1a, arrow) which results from association with filamentous structures. By contrast, mitochondria are oval or elongated and appear to be concentrated in the perinuclear region of the cell (Fig. Ib). When the cells are extracted with Triton X-100 $(0.5 \%, 5 \mathrm{~min})$, both organelles remain with the resulting cytoskeleton (Fig. Ic, d). The appearance and distributions of both structures are similar to those seen in the living cells indicating that the extraction procedure has not produced redistribution of either droplets or mitochondria, although some mitochondria have been broken into smaller segments by treatment with Triton X-100 (Fig. 1d). The extraction procedure has cleared the cells of cytoplasm and other structures so that the cytoskeletons show higher. resolution of the stained structures (Fig. Ic, d).

Double indirect immunofluorescence was used to reveal lipid droplets and vimentin-intermediate filaments or mitochondria and vimentin-intermediate filaments (Fig. 2a, b and c, d, respectively). The relationship between droplets and vimentinintermediate filaments is shown (Fig. 2a). Droplets are seen only in proximity to the filaments and careful examination shows that individual droplets are seen along the course of a given filament. With the blue filter, the droplets are clearly identified by shape and can be distinguished from the accompanying filaments (Fig. 2a). Only the lipid droplets can be seen when the sample is viewed with the green filter (Fig. 2b). Mitochondria are seen to be distributed along the course of intermediate filaments suggesting an association between these structures (Fig. $2 c$, d). These associations are seen at high magnification (Fig. 3) 

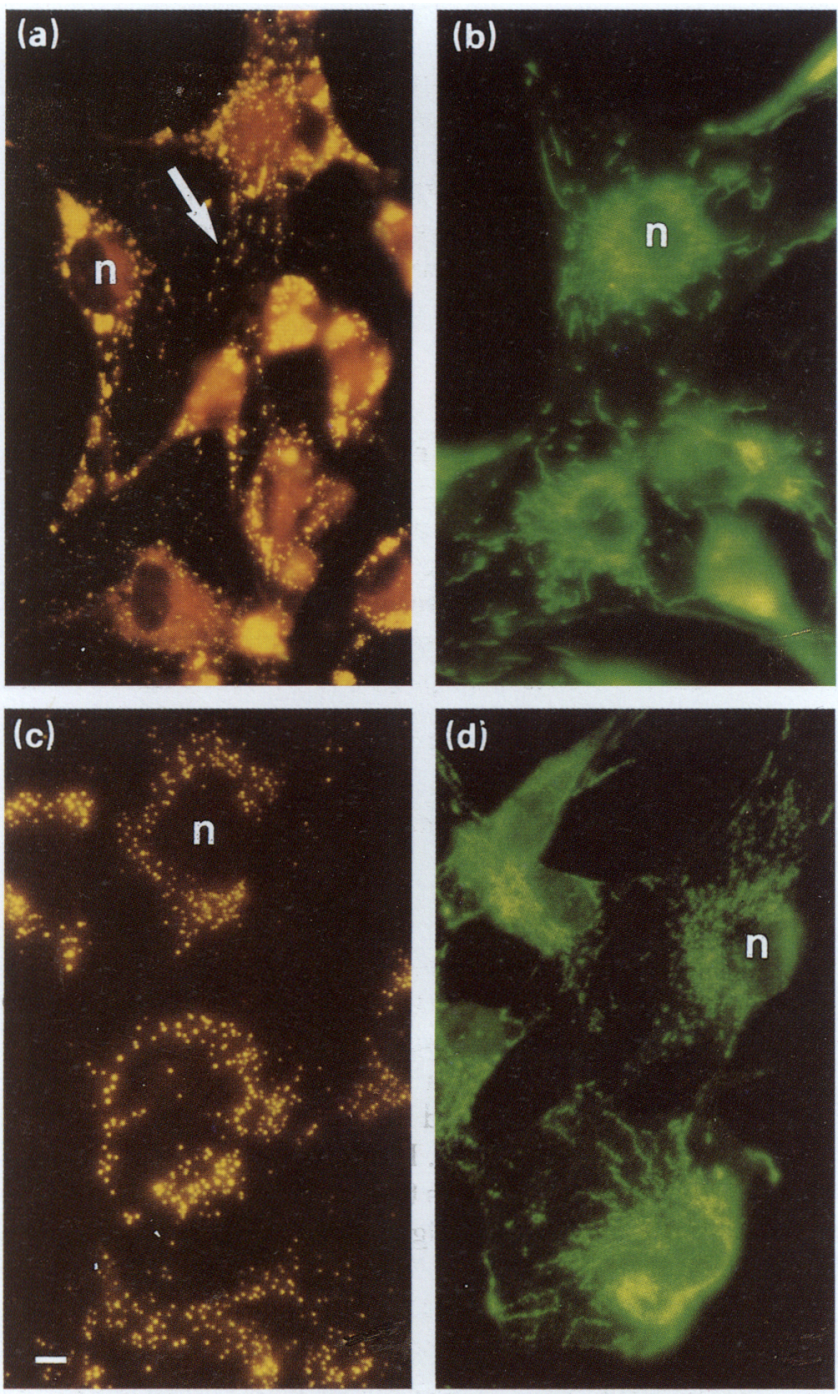

Fig. 1. Fluorescence and immunofluorescence staining of living and extracted Leydig cells. (a) and (b); living cells; (c) and (d): extracted cells $(0.5 \%$ Triton, $5 \mathrm{~min}$ ). In (a) and (c) lipid droplets are stained with Nile red; in (b) mitochondria are stained with rhodamine 123 and in (d) mitochondria labelled with anti-cytochrome oxidase and second antibody conjugated to FITC. Arrow indicates lipid droplets arranged in linear array; $\mathrm{n}$ : nucleus. $\mathrm{Bar}=5 \mu \mathrm{m}$.

which shows fields from cells treated as for Fig. 2a and c. It is clear that droplets are distributed along the course of filaments and that mitochondria are superimposed upon filaments so that the two structures are not distinctly separated (arrows). The co-localization of droplets and mitochondria with intermediate filaments is sufficiently strong to withstand extraction with detergent and extensive washing. It should be noted that lipid droplets and mitochondria are seen only in those regions of the cells in which vimentin-intermediate filaments are present. This excludes the possibility that co-localization between these organelles and intermediate filament is due simply to nonspecific crowding of intracellular contents. Co-localization between droplets and mitochondria on the one hand and microfilaments or microtubules on the other hand was never observed.

\section{Electron microscopy}

Intact cells. Transmission electron microscopy of a single rat Leydig cell is shown (Fig. 4). The most prominent organelles are lipid droplets and mitochondria. In addition, the usual organelles are present. The appearance and distribution of lipid droplets and mitochondria throughout the cell are consistent with the findings with light microscopy described above. The upper inset shows numerous intermediate filaments that make direct contact with lipid droplets. The lower inset shows similar contact between intermediate filaments and mitochondria. The diameters of these filaments were found to be $10.0 \pm 2 \mathrm{~nm}$ (mean and range, $n=100$ ) which is the accepted range for intermediate filaments. This cell is representative of many Leydig cells subjected to thin section without any extraction procedure.

Extracted cells. When the Leydig cells were extracted with Triton X-100 under mild conditions (0.5\% v:v for $5 \mathrm{~min})$ cytosol was removed from the cells (Fig. 5a). Lipid droplets, mitochondria and endoplasmic reticulum are seen. However, the extraction procedure damaged the outer mitochondrial membrane so that these organelles did not retain their usual morphology. The inner structures of these organelles have rounded up into spherical structures; the endoplasmic reticulum is fragmented and dilated, but the extraction procedure has not changed the appearance of lipid droplets.

More extensive extraction ( $1 \%$ Triton for $10 \mathrm{~min}$, then $1 \%$ Triton plus ammonium sulfate, for $10 \mathrm{~min}$ ) of the Leydig cells results in a preparation of intermediate filaments from which the remaining cytoskeletal elements have been removed. Under these conditions, lipid droplets remain associated with the filaments and all other cellular structures, including mitochondria, have been removed (Fig. $5 \mathrm{~b}$ ). The nuclear skeleton can be seen in such preparations (Fig. 5b). It should be noted that the various extraction procedures from mild to severe do not release increasing proportions of all internal structures but are selective in what is released. For example, plasma membrane can be largely removed by very mild extraction. More severe extraction leaves mostly mitochondria, droplets and filaments. Finally, with severe extraction, only droplets and intermediate filaments remain. Some of these stages are illustrated here (Figs 5a, b, 6 and 7). Clearly mitochondria and lipid droplets are not nonspecifically trapped in a meshwork of intermediate filaments.

Contact sites. Stereo pairs were prepared from severely extracted Leydig cells to demonstrate direct contact between filaments and lipid droplets (Fig. 6a, b). With the stereoscope, it is possible to see that some filaments end in contact with the droplet and hence both structures are located in the same plane within the thickness of the section (arrows). Similar studies were performed to show direct contact between filaments and mitochondria in mildly extracted cells (Fig. 7a, b, arrows).

\section{Immunoelectron microscopy}

The distribution of gold particles $(5 \mathrm{~nm})$ in non-osmicated preparations treated with anti-vimentin is shown (Fig. 8a). Gold particles are seen along the course of $10 \pm 2 \mathrm{~nm}$ filaments (mean and range, $n=100$ ). Non-osmicated preparations display a high contrast between the gold particles and the 

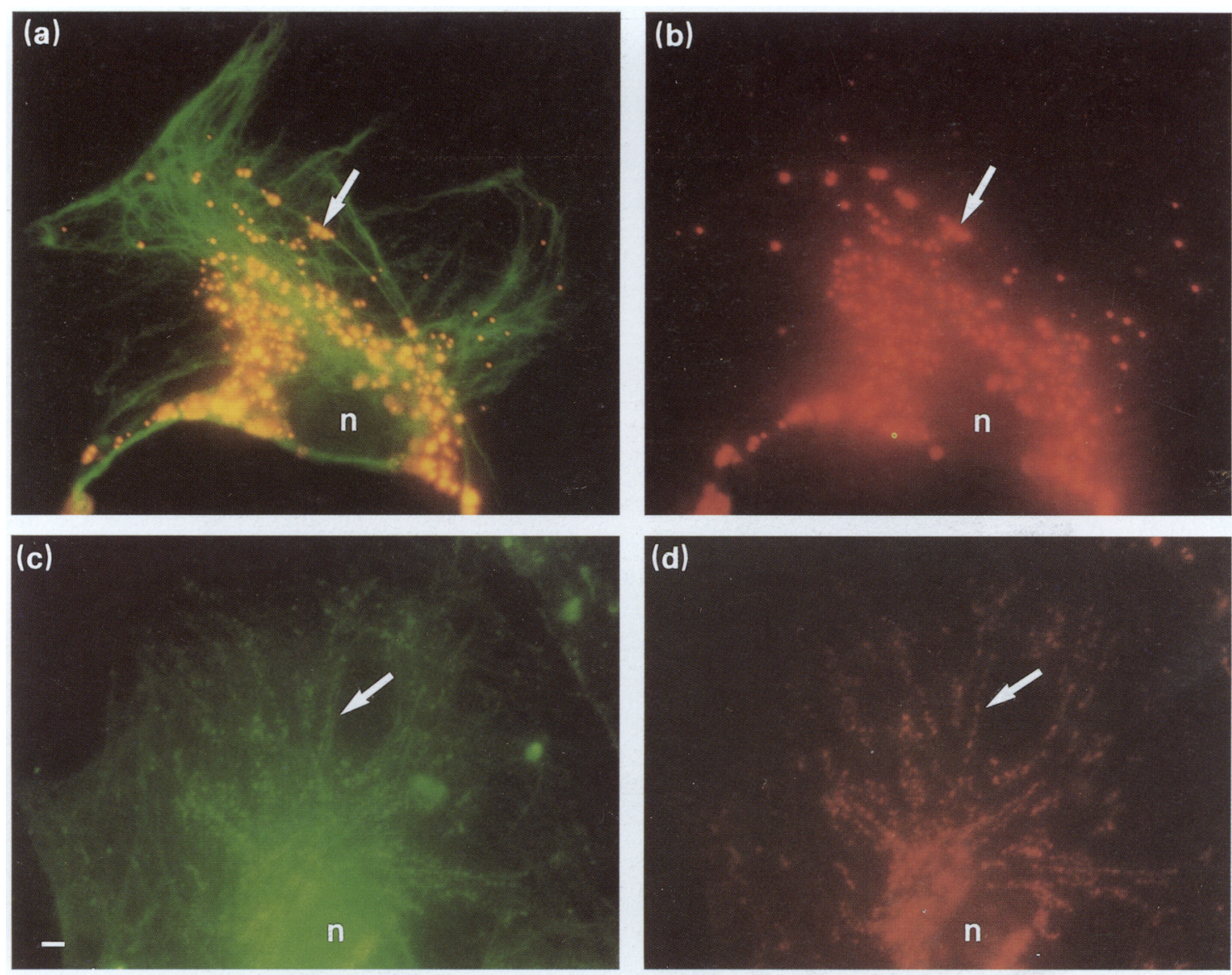

Fig. 2. Double staining of extracted ( $0.5 \%$ Triton, $5 \mathrm{~min}$ ) Leydig cells. (a) Lipid droplets and vimentin-intermediate filaments; (b) lipid droplets of the same cell as in (a); (c) mitochondria and vimentin intermediate filaments; (d) mitochondria of the same cell as in (c). (a) and (c): blue filter; (b) and (d): green filter. Arrows indicate lipid droplets (a, b) or mitochondria (c, d). n: nucleus. Bar $=2 \mu \mathrm{m}$.
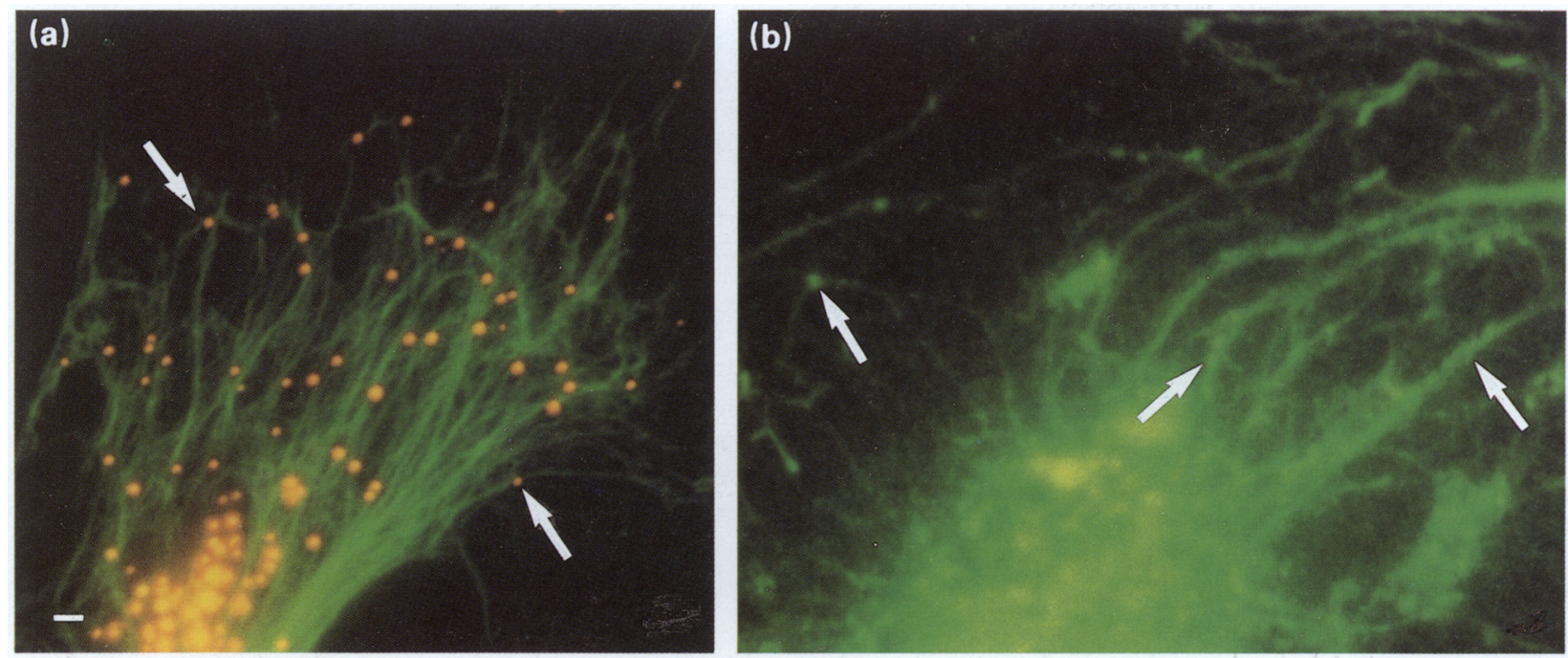

Fig. 3. High magnification of double staining of extracted (0.5\% Triton, 5 min) Leydig cells. (a) Lipid droplets and vimentin-intermediate filaments; (b) mitochondria and vimentin-intermediate filaments. Arrows indicate (a) lipid droplets or (b) mitochondria. Bar $=1.4 \mu \mathrm{m}$.

underlying filaments. From our experience, intermediate filaments become very darkly stained after treatment with osmium tetroxide. This largely reduces the contrast and, therefore, obscures the visibility of the gold particles. When no first antibody (Fig. 8b) or normal mouse serum were used, no gold particles were seen. In considering the diameters of intermediate 


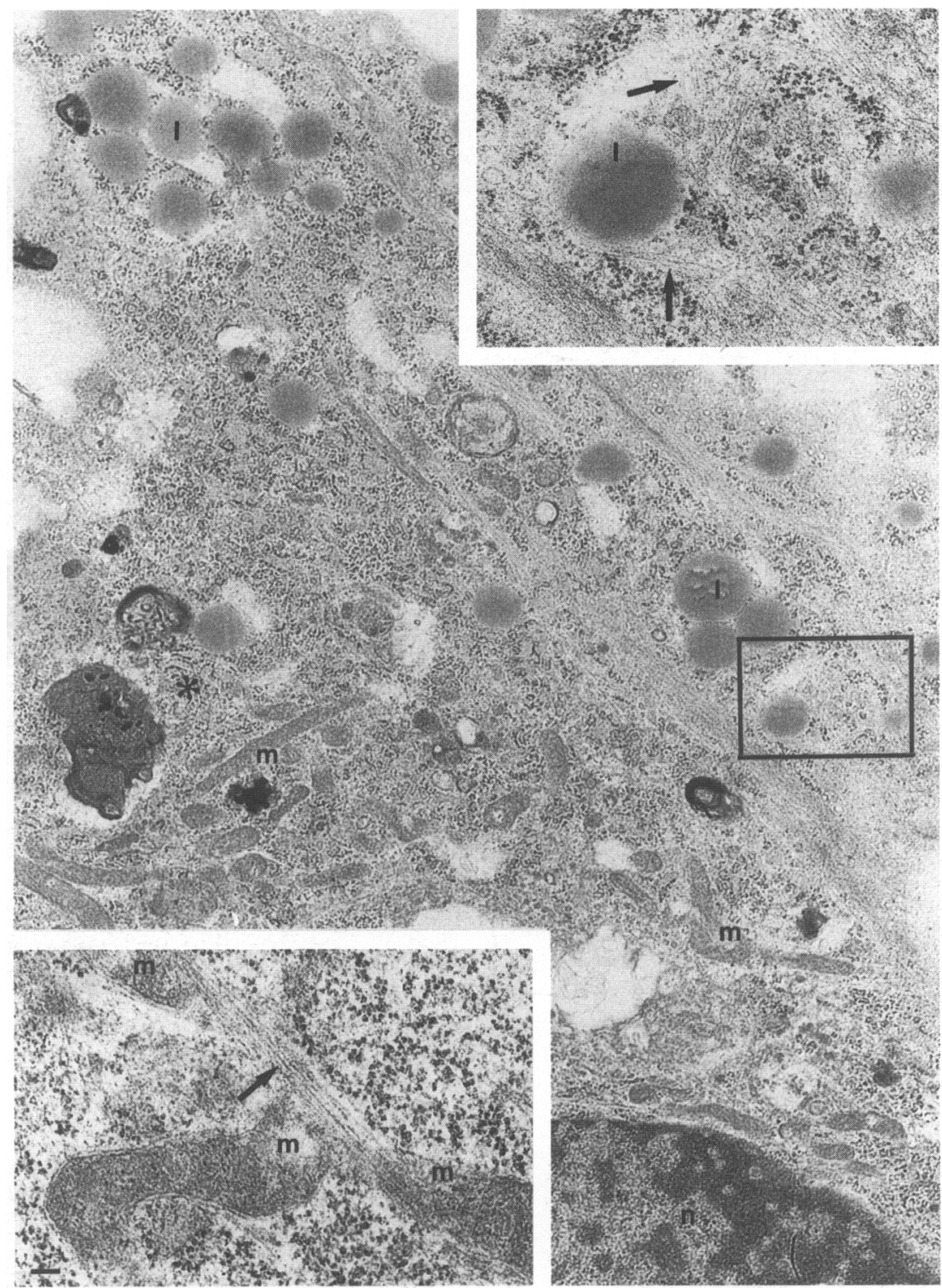

Fig. 4. Electron microscopy of intact Leydig cells. Low magnification of a Leydig cell in primary culture is shown. Upper inset: high magnification of the area limited by a rectangle in the main micrograph showing the direct contact between intermediate filaments and lipid droplets. Lower inset: direct contact between intermediate filaments and mitochondria. Arrows: intermediate filaments; *: endoplasmic reticulum; I: lipid droplet; m: mitochondria; n: nucleus. Bar $=0.3 \mu \mathrm{m}$ (main), $0.1 \mu \mathrm{m}$ (insets).

filaments, it is necessary to consider at least three factors that may influence the apparent diameters of these structures. First, intermediate filaments may associate with each other in bundles. Second, the process of shadowing leaves a layer of the shadowing material along the course of some filaments. Third, the preparation of intermediate filaments may result in the deposition of a proteinaceous material of unknown origin along the filaments. Careful examination will reveal the true intermediate filament as a dark core within the coated structure especially in osmicated preparation (Fig. 8 b). These factors were all taken into consideration in estimating the true diameter of the structures in our preparations as $10 \pm 2 \mathrm{~nm}$. In this connection it should be noted that the gold particles indicating the presence of anti- vimentin (and hence vimentin itself) are confined to the central region of the filaments.

\section{Discussion}

The present studies are based upon primary cultures of rat Leydig cells, the ultrastructure of which has not been reported. It is known that rat Leydig cells in culture retain their normal steroidogenic activity for up to two (Rommerts et al., 1982) or three (Mather et al., 1981) days and then production of androgens starts to decline. Our findings confirm this conclusion as judged by the production of testosterone with and without 

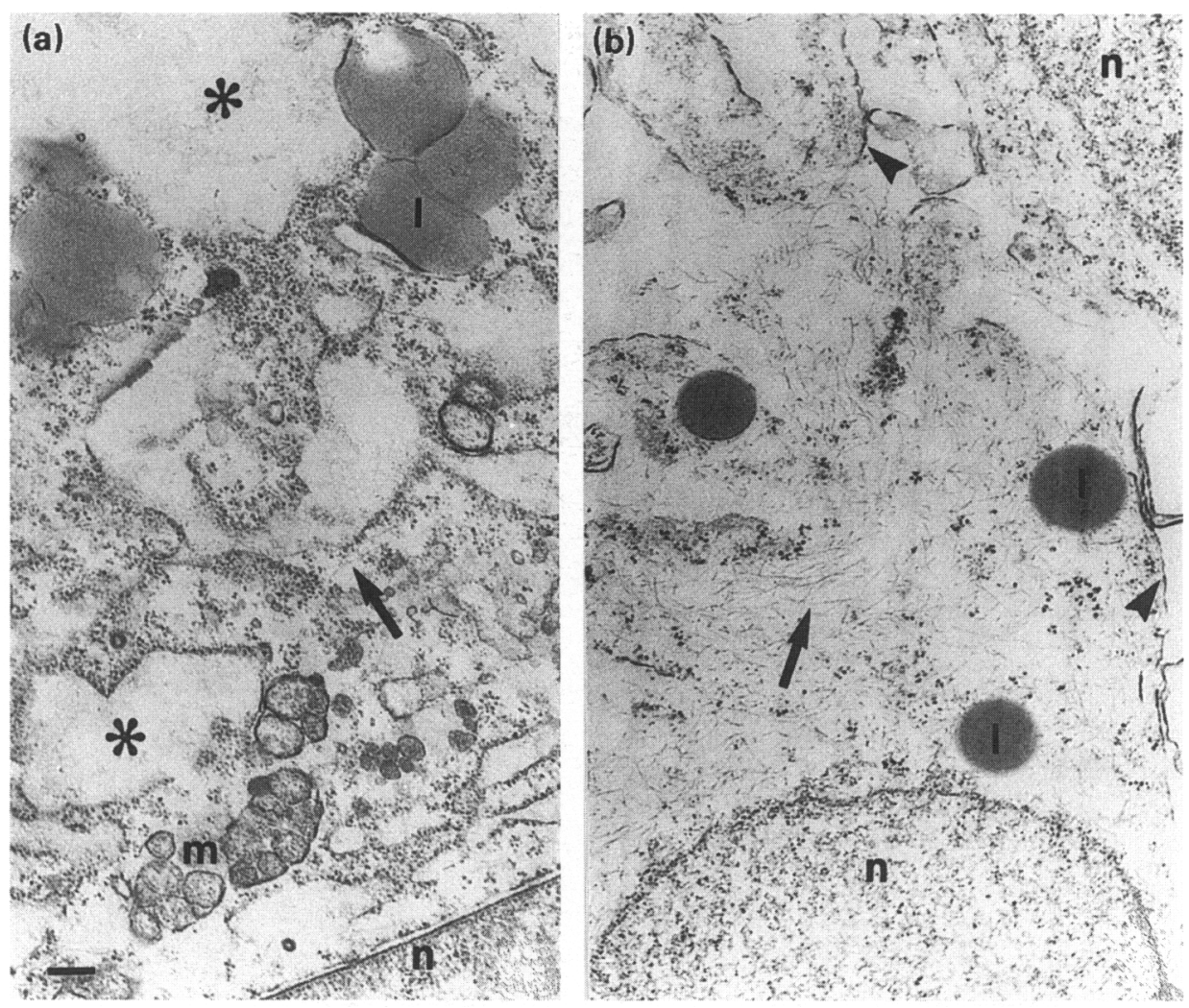

Fig. 5. Electron microscopy of extracted cells. (a) Mild extraction ( $0.5 \%$ Triton, $5 \mathrm{~min})$, and (b) intermediate filaments preparation ( $1 \%$ Triton, $10 \mathrm{~min}$ then $1 \%$ Triton plus ammonium sulfate, $10 \mathrm{~min}$ ). Arrow: intermediate filaments; arrowhead: residual cell membrane; $*$ : dilated endoplasmic reticulum; I: lipid droplet; $\mathrm{m}$ : mitochondria; $\mathrm{n}$ : nucleus. $\mathrm{Bar}=0.25 \mu \mathrm{m}$.
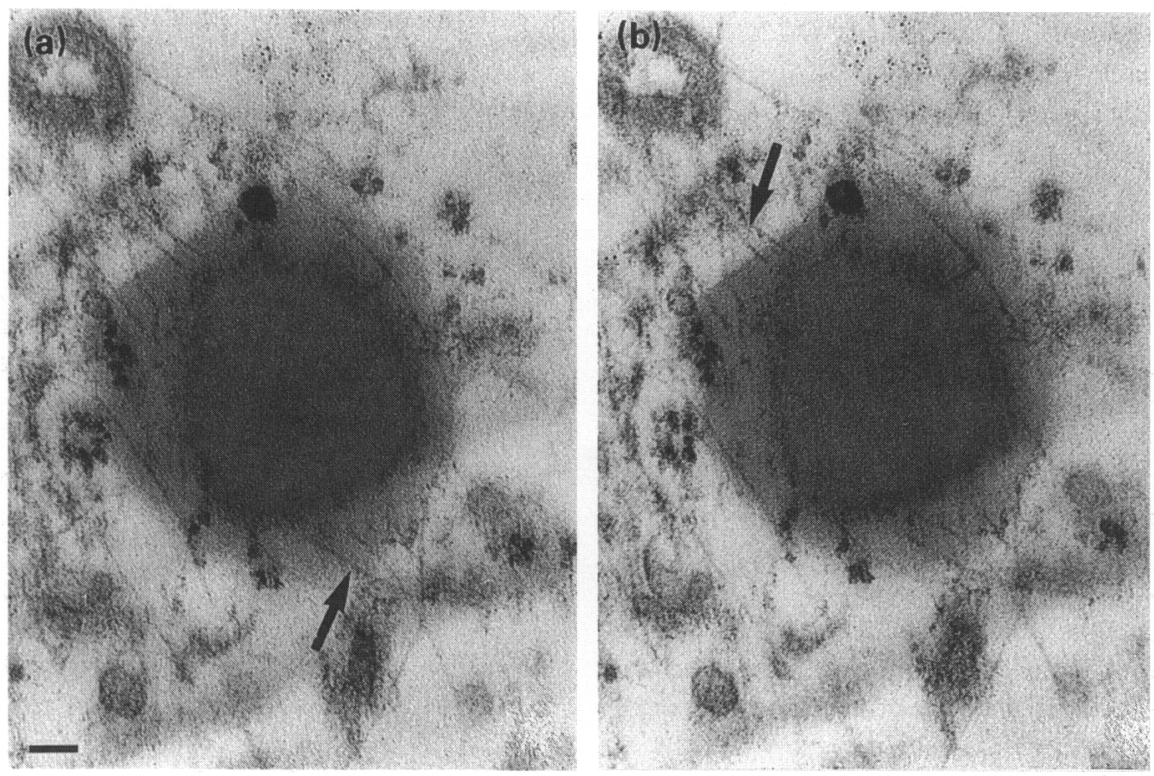

Fig. 6. Stereo pair electron microscopy of severely extracted (1\% Triton, $10 \mathrm{~min}$ ) Leydig cells. Arrows indicate the intermediate filaments that are in direct contact with a lipid droplet photographed at (a) -12 and (b) +12 degrees of tilting. Bar $=0.08 \mu \mathrm{m}$. 

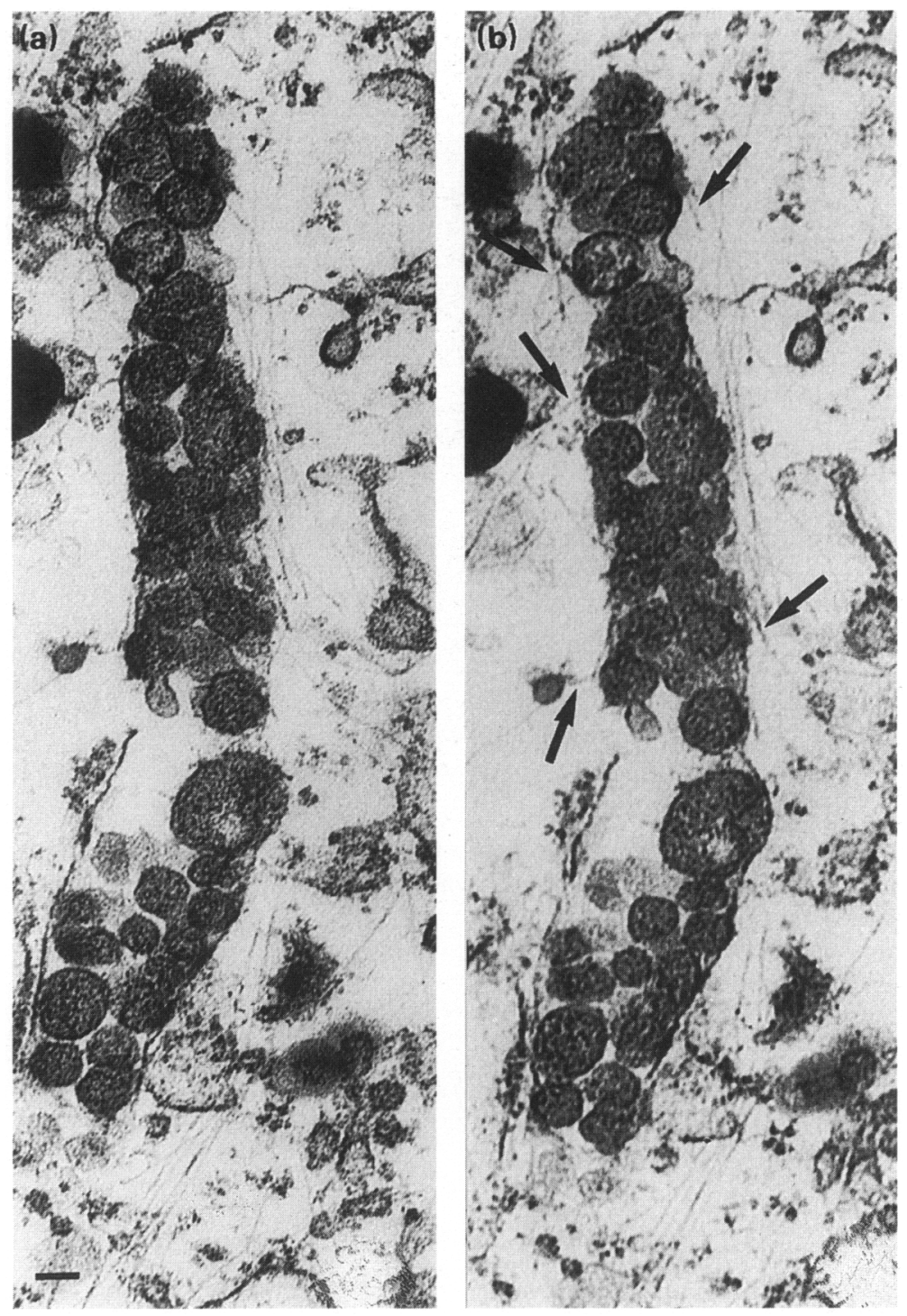

Fig. 7. Stereo pair electron microscopy of Leydig cells after mild extraction $(0.5 \%$ Triton, $5 \mathrm{~min}$ ). Arrows indicate the contact points between intermediate filaments and a mitochondrion photographed at (a) -12 and (b) +12 degrees of tilting. Bar $=0.08 \mu \mathrm{m}$.

dibutyryl cAMP. The ultrastructure of the cells shown after 3 days in culture reveals that the cells and their steroidogenic organelles are very similar to those of cultured adrenal cells (Almahbobi et al., 1992a, b). The structures described here as vimentin-intermediate filaments have been identified by three criteria. First, in extracted cells, filamentous structures reacting with anti-vimentin were seen. Second, structures of similar appearance and distribution were of the diameter characteristic of intermediate filaments i.e. $10 \pm 2 \mathrm{~nm}$ (means and range, $n=100$ ). Third, these structures were identified by immunoelectron microscopy. Moreover, the intermediate filaments were prepared by a method known to yield only these structures in other cells (Fey et al., 1984).

In these studies we have investigated the association between two intracellular structures necessary for steroid synthesis, namely lipid droplets and mitochondria with vimentin- intermediate filaments. Cholesterol is stored as ester in lipid droplets and must be cleaved to give free cholesterol which is transported to the inner mitochondrial membrane where steroid synthesis begins. This process of transport is of particular importance because it involves the rate-limiting step in steroid synthesis - one that is specifically stimulated by LH and ACTH (Hall et al., 1979; Osawa et al., 1984; Hall, 1987). The process is poorly understood but is known to involve the cytoskeleton (Hall et al., 1979; Osawa et al., 1984; Hall, 1987). It is, therefore, of considerable interest to learn that the droplets are tightly attached to intermediate filaments. In attempting to explain the resistance of the lipid content of the droplets to detergents and other agents used in electron microscopy, we found that in adrenal cells the lipid is protected by a capsule containing vimentin (Almahbobi and Hall, 1990; Almahbobi et al., 1992a). It seems likely that the same protection of droplets occurs in Leydig cells 

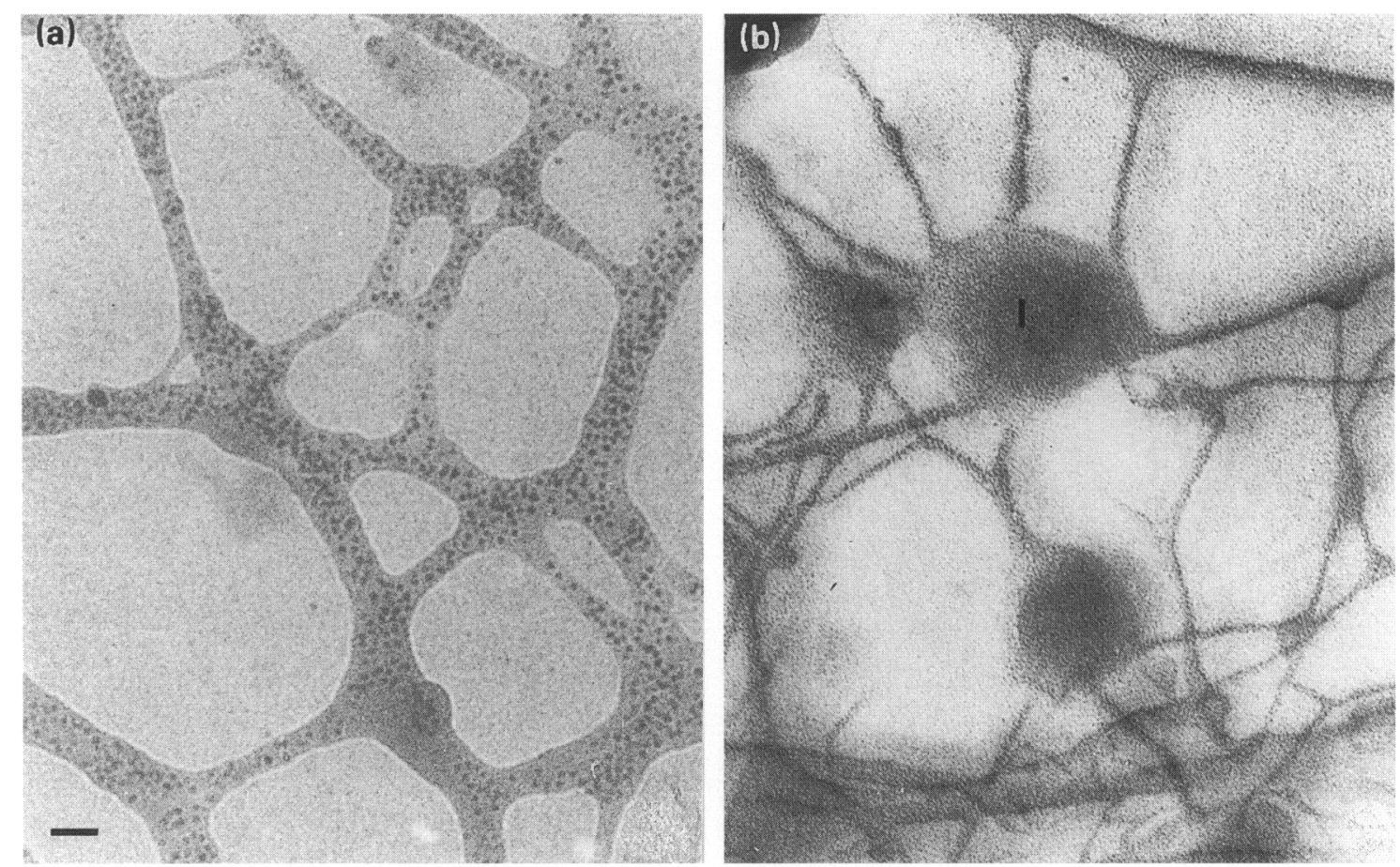

Fig. 8. Immunoelectron microscopy of intermediate filaments preparation ( $1 \%$ Triton, $10 \mathrm{~min}$ then $1 \%$ Triton plus ammonium sulfate, $10 \mathrm{~min}$ ) of cultured Leydig cells. The filaments were prepared as whole mounts and were stained with (a) anti-vimentin antibody followed by secondary antibody conjugated to gold particles $(5 \mathrm{~nm})$. Although filaments appear thicker than the normal diameter in such a preparation, gold particles are seen along the central part (10 $\pm 2 \mathrm{~nm}$ ) of the filaments. This preparation was not post-fixed with osmium tetroxide. (b) Control preparation with no first antibody. This preparation was treated with osmium tetroxide. l: lipid droplet. $B a r=0.05 \mu \mathrm{m}$.

which clearly resist the same treatments. As in adrenal cells, the droplets are 0.2 to $3 \mu \mathrm{m}$ in diameter and are usually roughly spherical (Almahbobi and Hall, 1990).

Attachment of lipid droplets to intermediate filaments was demonstrated in Leydig cells by several methods. First, in immunofluorescence, lipid droplets are seen along the course of filaments and not in regions of the cell where there are no filaments. Second, electron microscopy of intact cells shows filaments apparently touching lipid droplets. This association is more obvious when cells are cleared by extraction with Triton X-100. Finally, direct contact between these structures can be seen in stereo pairs with tilting. This approach offers the most convincing evidence of direct contact because it provides a three-dimensional image of the structures in question. In nonsteroidogenic cells, tight association between lipid droplets and intermediate filaments has been reported in breast adipocytes (Franke et al., 1987).

Attachment of mitochondria to intermediate filaments is strongly suggested by the appearance of the mitochondria along the course of the filaments. Mitochondria are not seen at a distance from filaments on immunofluorescence. The apposition of elongated mitochondria with filaments results in the appearance of a composite structure consisting of filaments and mitochondria in close association. Transmission electron microscopy also shows close association between these structures and, once again, direct contact is established by means of stereo pairs. Close association between intermediate filaments and mitochondria has been reported in Leydig cells (David-Ferreira and
David-Ferreira, 1980), in hepatocytes (Fiskum et al., 1980), in HeLa cells and aortic smooth muscle cells (Lin et al., 1990) and in fibroblasts (Mose-Larsen et al., 1982).

Extraction of Leydig cells with Triton does not disturb the attachment of droplets to intermediate filaments but destroys the outer mitochondrial membrane and, hence, releases mitochondria from the filaments. However, indirect immunofluorescence with anti-cytochrome oxidase following such extraction reveals some traces of fluorescent staining, probably associated with intermediate filaments, suggesting that mitochondria may be tightly attached to intermediate filaments (not shown). This finding was also made with adrenal cells (Almahbobi et al., 1992b). This means that the attachment of mitochondria to filaments is not less tenacious than that between droplets and filaments but rather that the mitochondrial membrane is susceptible to the action of Triton.

Since the first step in the conversion of cholesterol to steroid hormones involves transport of that substrate to mitochondria and since the cytoskeleton is required for this process, the attachment of both the source of cholesterol (droplets) and the site of its conversion to pregnenolone (mitochondria) to the same filamentous component of the cytoskeleton is likely to be of functional importance. Earlier reports in the adrenal cortex show droplets in close physical contact with mitochondria (Merry, 1975). Moreover, evidence for an association between vimentin-intermediate filaments and smooth endoplasmic reticulum has been reported in Leydig cells of rat testicular tissue (Russell et al., 1987). The fact that the three main steroidogenic organelles (lipid droplets, mitochondria and endoplasmic 
reticulum) are associated with vimentin-intermediate filaments highlights the significance of these findings. Indeed, as Steinert and Liem (1990) point out, intermediate filaments far from being inert, supporting structures are extremely dynamic, and participate in numerous cellular activities. Vimentin-intermediate filaments play a functional role in the intracellular transport of lipoprotein-derived cholesterol in human SW-13 adrenal tumour cells (Sarria et al., 1992).

Studies from this laboratory (Hall et al., 1979; Osawa et al., 1984; Hall, 1987) showed that actin microfilaments are required for the increase in cholesterol transport produced by $\mathrm{LH}$ and ACTH. A combination of some contractile event involving actin and the attachment of droplets and mitochondria to intermediate filaments may provide the elements necessary to regulate the transport of cholesterol by movement of droplets to mitochondria or vice versa. In canine interstitial cells, filaments $(5 \mathrm{~nm}$ in diameter) of unknown composition were reported to occur near lipid droplets suggesting that these filaments could be involved in cholesterol transport (Connell and Christensen, 1975). The fact that both adrenal and Leydig cells show the same cellular organization for cholesterol transport is consistent with the fact that steroid synthesis appears to be organized in a similar fashion in all steroidogenic cells.

These studies were supported by NH and MRC grant no. 900706 . The authors are grateful to the staff of Electron Microscopy Unit at the University of New South Wales for the use of their equipment.

\section{References}

Almahbobi G and Hall PF (1990) The role of intermediate filaments in adrenal steroidogenesis Journal of Cell Science 97 679-687

Almahbobi G and Hall FP (1993) Indirect immunofluorescence modified to display two antigens with one light filter Histochemical Journal 25 14-18

Almahbobi G, Silberzahn N, Fakhri R and Silberzahn P (1985) Steroidogenic characteristics of the adrenal cortex of the mare studied by electron microscopy Archive Anatomie Microscopique Morphologie Experimentale 74 193-203

Almahbobi G, Papadopoulos V, Carreau S and Silberzahn P. (1988) Age-related morphological and functional changes in the Leydig cells of the horse. Biology of Reproduction 38 653-665

Almahbobi G, Williams LJ and Hall PF (1992a) Attachment of steroidogenic lipid droplets to intermediate filaments in adrenal cells Journal of Cell Science 101 383-393

Almahbobi G, Williams LJ and Hall PF (1992b) Attachment of mitochondria to intermediate filaments in adrenal cells: relevance to the regulation of steroid synthesis Experimental Cell Research $200361-369$

Browning JY, D'Agata R and Grotjan HE, Jr (1981) Isolation of purified rat Leydig cells using continuous Percoll gradients Endocrinology 109667-669
Connell CJ and Christensen AK (1975) The ultrastructure of the canine testicular interstitial tissue Biology of Reproduction 12 368-382

David-Ferreira KL and David-Ferreira JF (1980) Association between intermediate sized filaments and mitochondria in rat Leydig cells Cell Biology Intemational Reports 4 655-662

Eytan GD, Carroll RC, Shatz G and Racker E (1975) Arrangement of the subunits in solubilized and membrane-bound cytochrome $\mathrm{C}$ oxidase from bovine heart Joumal of Biological Chemistry $2508598-8603$

Fey EG, Wan KM and Penman S (1984) Epithelial cytoskeletal framework and nuclear matrix-intermediate filament scaffold: three dimensional organisation and protein composition Journal of Cell Biology 98 1973-1984

Fiskum G, Craig SW, Decker GL and Lehninger AL (1980) The cytoskeleton of digitonin-treated rat hepatocytes Proceedings National Academy of Sciences USA 77 3430-3434

Franke WW, Hergt M and Grund C (1987) Rearrangement of vimentin cytoskeleton during adipose conversion; formation of an intermediate filament cage around lipid globules Cell 49 131-141

Greenspan P, Mayer EP and Fowler SD (1985) Nile red: a selective fluorescent stain for intracellular lipid droplets Journal of Cell Biology 100 965-973

Hall PF (1987) ACTH and corticosteroidogenesis. In Hormonal Proteins and Peptides, Vol. XIII, pp 89-125. Academic Press, NY

Hall PF, Charponnier C, Nakamura M and Gabbiani G (1979) The role of microfilaments in the response of Leydig cells to luteinising hormone Journal of Steroid Biochemistry 11 1361-1366

Johnson LV, Walsh ML and Chen LB (1980) Localisation of mitochondria in living cells with Rhodamine 123 Proceedings National Academy of Sciences USA 77 990-994

Lin A, Krockmalnic G and Penman S (1990) Imaging cytoskeleton-mitochondrial membrane attachment by embedment-free electron microscopy of saponinextracted cells Proceedings National Academy of Sciences USA 87 8565-8569

Mather JP, Saez JM and Haonr F (1981) Primary cultures of Leydig cells from rat, mouse and pig: advantages of porcine cells for the study of gonadotropin regulation of Leydig cell function Steroids 38 35-42

Merry J (1975) Mitochondrial structure in the adrenal cortex Joumal of Anatomy $119611-618$

Mose-Larsen P, Bravo R, Fey SJ, Small JV and Celis JE (1982) Putative association of mitochondria with a subpopulation of intermediate-sized filaments in cultured human skin fibroblasts Cell 31 681-692

Osawa S, Betz G and Hall PF (1984) The role of actin in the responses of adrenal cells to ACTH and cyclic AMP: inhibition of DNase Journal of Cell Biology 99 1335-1342

Rommerts FFG, Roemburg van MJA, Lindh LM, Hegge JAJ and van der Molen HJ (1982) The effects of short-term culture and perfusion on LH-dependent steroidogenesis in isolated rat Leydig cells Joumal of Reproduction and Fertility $65 \quad 289-297$

Russell LD, Amlani SR, Vogl AW and Weber JE (1987) Characterisation of filaments within Leydig cells of the rat testis American Journal of Anatomy 178 $231-240$

Sarria AJ, Panini SR and Evans RM (1992) A functional role for vimentin intermediate filaments in the metabolism of lipoprotein-derived cholesterol in human SW-13 cells Joumal of Biological Chemistry $26719455-19463$

Silberzahn P, Almahbobi G, Dehennin L and Meroune A (1985) Estrogen metabolites in equine ovarian follicles: a chromatographic-mass spectrometric determination in relation to follicular ultrastructure and progestin content Joumal of Steroid Biochemistry 22 501-505

Steinert PM and Liem RKH (1990) Intermediate filaments dynamics Cell 60 $521-523$ 